\title{
Digital SyNTHESIS OF ANALOG SignAlS FOR Audiometric APPliCations
}

\author{
S. Brigati ${ }^{(1)}$, F. Francesconi $^{(1)}$, P. Malcovati ${ }^{(2)}$, M. Poletti $^{(2)}$ and F. Maloberti $^{(2)}$ \\ (1) Micronova Sistemi S.r.l., Via Volta 12, 27100, Pavia, Italy \\ Tel. +39382 34626, Fax. +39382303653 \\ (2) Integrated Microsystem Laboratory, University of Pavia, Via Ferrata 1, 27100, Pavia, Italy \\ Tel.+39382505 205, Fax.+39382505677, E-Mail: piero@ipvsp4.unipv.it
}

\begin{abstract}
This paper presents a novel signal generator, based on the direct digital synthesis (DDS) technique, which fulfills the requirements of advanced audiometric systems. The described solutions face two different problems, namely the generation of a finely tunable pure sinewave and the generation of noise signals with controlled spectrum. In order to achieve tuning capabilities down to $1 \mathrm{~Hz}$ at $20 \mathrm{kHz}$ or $15 \mu \mathrm{Hz}$ at $100 \mathrm{~Hz}$, a fractional division of a $40 \mathrm{MHz}$ master clock with noise shaping of the resulting error is performed. Moreover, for noise generation a novel circuit based on pseudo-random sequences combined with analog SC filters is used.
\end{abstract}

\section{INTRODUCTION}

Medical instruments for ear, nose, and throat (ENT) such as audiometers, impedancemeters, evoked potential systems and otoemission systems are becoming more demanding in terms of audio signal quality. In all of these instruments, in fact, audio signals, such as pure sinewaves with variable frequency (from 125 to $20000 \mathrm{~Hz}$ ), narrow band noise, speech noise and white noise are required, as shown in Fig. 1.

Nowadays, thanks to the availability of mixed ana$\log$ /digital IC technologies, several functions which were typically implemented with critical analog circuits can be more comfortably implemented using digital techniques. Examples are pure tone generation which can enjoy the benefits of direct digital synthesis (DDS) [1] and white noise generation, which can exploit pseudo-random digital circuits.

One of the most critical requirements for sinewave generators is the stability and the resolution of the obtained frequency. Traditional analog sinusoidal sources allow a resolution of about $5 \mathrm{~Hz}$ to be achieved over the audio band. However, in the new generation of audio instruments, resolutions lower than $1 \mathrm{~Hz}$ are required. To fulfill these specifications, therefore, an analog solution would require extremely precise and stable components, or online trimming and tuning, thus increasing the cost of the system. By contrast, the performance of a digital solution

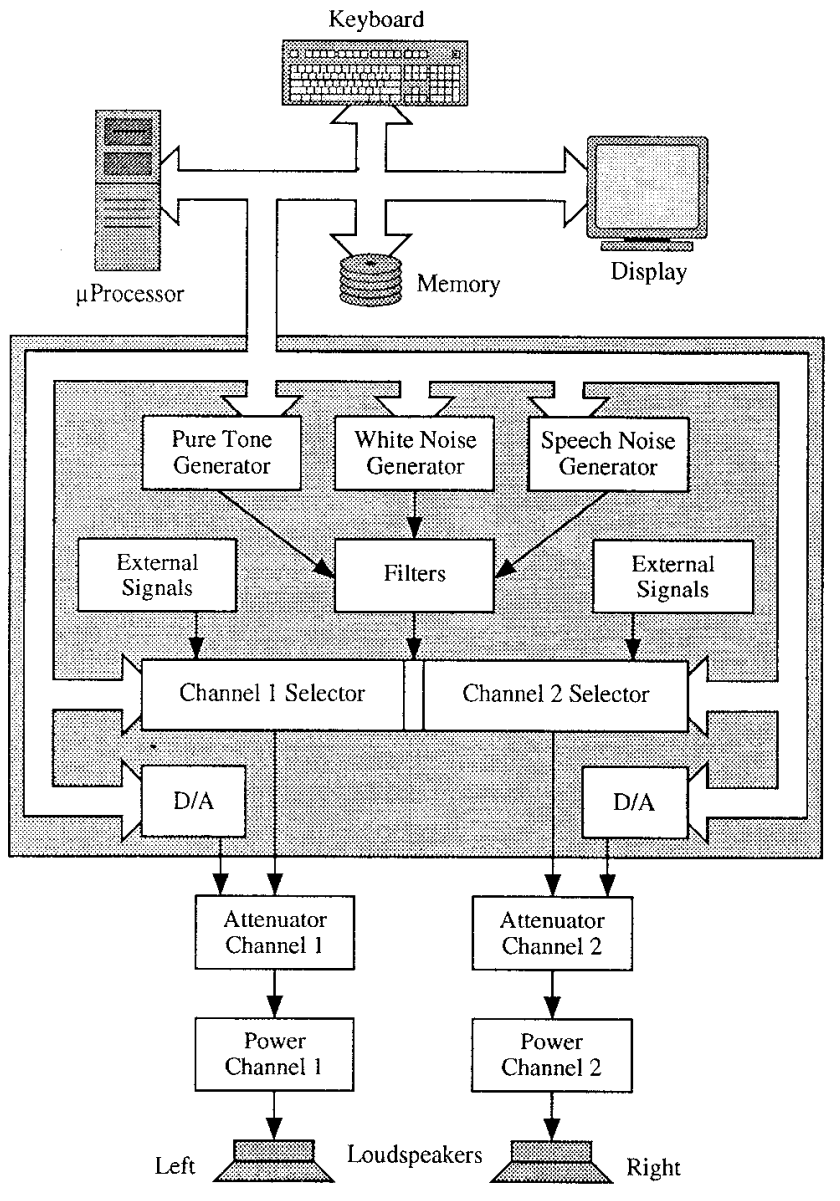

Fig. 1 - Block diagram of a typical audiometer

is determined by very stable clock signals available at low cost.

Similar considerations can be applied to white noise generators. Analog solutions are typically based on highvalue resistors, which are very sensitive to interferences and require on-line calibration of temperature effects. By contrast, pseudo-random sequence generators (PRSG) allow a perfectly white and repeatable spectrum to be obtained with very simple hardware.

In this paper we present novel architectures for DDS and PRSG to be used in high performance medical instruments. The most important specifications of the system are summarized in Tab. 1 [2]. 


\begin{tabular}{|l|c|}
\hline \multicolumn{1}{|c|}{ Parameter } & Value \\
\hline Master Clock Frequency & $40 \mathrm{MHz}$ \\
\hline Frequency Precision & $1 \%$ \\
\hline $\begin{array}{l}\text { Sinewave Frequency Range } \\
\text { (Pure Tone) }\end{array}$ & $100-20000 \mathrm{~Hz}$ \\
\hline Sinewave Resolution & $1 \mathrm{~Hz}$ \\
\hline Sinewave Warble & $-10 \%$ \\
\hline Sinewave Signal-to-Noise Ratio & $60 \mathrm{~dB}$ \\
\hline Total Harmonic Distortion & $-60 \mathrm{~dB}$ \\
\hline $\begin{array}{l}\text { White Noise Band } \\
\text { (F }=32 \text { kHz) }\end{array}$ & $16-16000 \mathrm{~Hz}$ \\
\hline White Noise Repetition Period & $>5 \mathrm{~s}$ \\
\hline White Noise Flatness & $\pm 5 \mathrm{~dB}$ \\
\hline
\end{tabular}

Tab. 1 - System specifications

In light of the extremely high accuracy and flexibility of the proposed digital signal processing circuits, the performance achieved is about 10 times better than in commercially available instruments with similar cost.

\section{Pure Tone Generator}

The basic principle of the proposed DDS system is illustrated in Fig. 2 [3, 4]. It consists of a programmable divider, a digital counter, a look-up table containing the digitized codes of a sinewave period (Sine ROM) and a digitalto-analog converter (DAC). The programmable division factor $(P D F)$ is provided to the system by an external input device, such as a microprocessor.

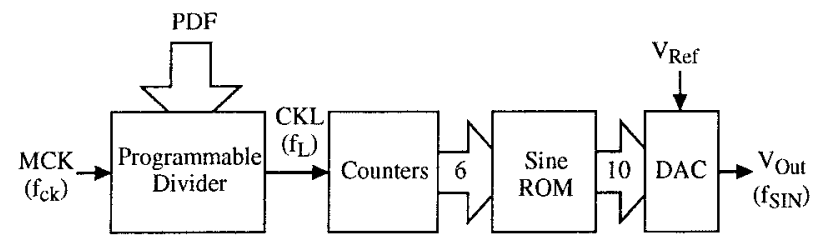

Fig. 2 - Basic principle of direct digital synthesis of sinusoidal signals

The detailed block diagram of the programmable divider is shown in Fig. 3. The master clock signal $M C K$ $\left(f_{c k}=40 \mathrm{MHz}\right)$ is accumulated by an $n_{P D I}$ bit synchronous counter. The resulting signal is compared during each master clock period with an $n_{P D I}$ bit reference word. When the two words become equal, the output signal of the divider $(C K L)$ is set to one and the synchronous counter is reset. The clock signal obtained ( $C K L)$ drives the input of a 6 bit counter, whose output word represents the address for the $6 \times 10$ bit look-up table containing a sampled sinewave period $\left(n_{\text {per }}=64\right.$ samples $/$ period $)$. The digital output code of the look-up table is finally converted into the analog domain by a 10 bit DAC.

The frequency of signal $C K L\left(f_{L}\right)$ obtained at the output of the programmable divider is given by

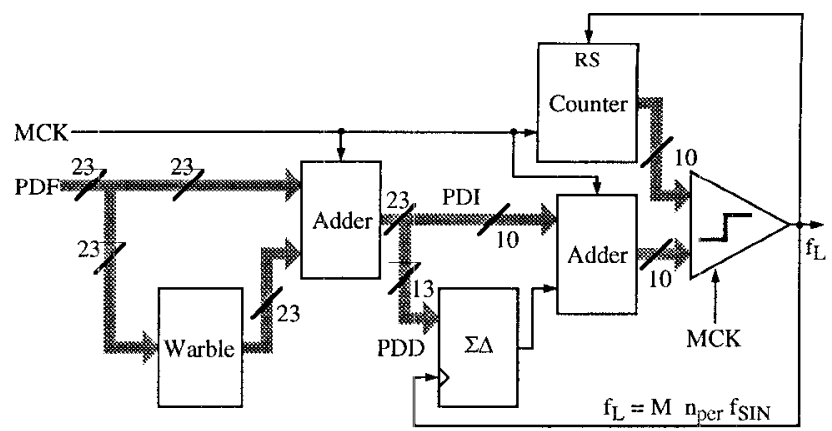

Fig. 3 - Block diagram of the programmable divider

$$
f_{L}=\frac{f_{c k}}{P D F} .
$$

The output sinewave frequency $\left(f_{S I N}\right)$, therefore, can be expressed as

$$
f_{S I N}=\frac{f_{L}}{n_{\text {per }} M},
$$

where $n_{p e r}$ denotes the number of sampled points of the sinewave period and $M$ is an additional division factor used to generate the clock signal for the switched-capacitor reconstruction filters required after the DAC.

Assuming $P D F$ to be an integer, the frequency resolution of the sinusoidal signal is limited by

$$
\Delta f=\frac{f_{c k}}{M n_{p e r}} \frac{1}{P D F(P D F+1)}=\frac{f_{S I N}^{2} n_{p e r} M}{f_{c k}+f_{S I N} n_{p e r} M} .
$$

In worst case conditions $\left(f_{c k}=40 \mathrm{MHz}, n_{p e r}=64, M=8\right.$ and $f_{S I N} \approx 20 \mathrm{kHz}$ ), in fact, we obtain $\Delta f=4076 \mathrm{~Hz}$, which is three orders of magnitude larger than the specifications.

Since the master clock frequency is already pretty high, it is not possible to fulfill the system specifications by further increasing the time discretization. Instead, we solved the problem by using non-integer division of the master clock period. The digital word $P D F$ actually consists of two coefficients: $P D I$ and $P D D$, which are the integer and fractional parts of the division factor, respectively. The wordlength of PDI $\left(n_{P D I}\right)$ is determined by the minimum sinewave frequency $(100 \mathrm{~Hz})$. Using Eqns. (1) and (2), we obtain

$$
n_{P D I}=\left.\ln _{2} \frac{f_{c k}}{n_{p e r} M f_{S I N}}\right|_{f_{S I N}=100 \mathrm{~Hz}} \cong 10 .
$$

By contrast, the wordlength of the fractional part $P D D$ $\left(n_{P D D}\right)$ is determined by the required resolution at the maximum sinewave frequency $(20 \mathrm{kHz})$, according to

$$
n_{P D D}=\left.\ln _{2} \frac{n_{p e r} M f_{S I N}\left(f_{S I N}-1\right)}{f_{c k}}\right|_{f_{S I N}=20 \mathrm{kHz}} \cong 13
$$

Therefore, the required wordlength of $P D F$ is 23 bits.

Achieving fractional division of the master clock period while keeping spurious signals produced by distortion and phase noise below $-60 \mathrm{~dB}$ is not a simple task. Therefore, 
in order to satisfy these requirements, we used oversampling and noise shaping techniques. In fact, the fractional component of $P D F(P D D)$ is applied at the input of a firstorder digital sigma-delta $(\Sigma \Delta)$ modulator, as shown in Fig. 3. The obtained bitstream is added to $P D I$. The actual division factor is, therefore, alternatively $P D I$ or $P D I+1$, according to the output of the $\Sigma \Delta$ modulator ( 0 or 1 ). The effect of the resulting frequency modulation of the clock signal $C K L$ is illustrated in Fig. 4.

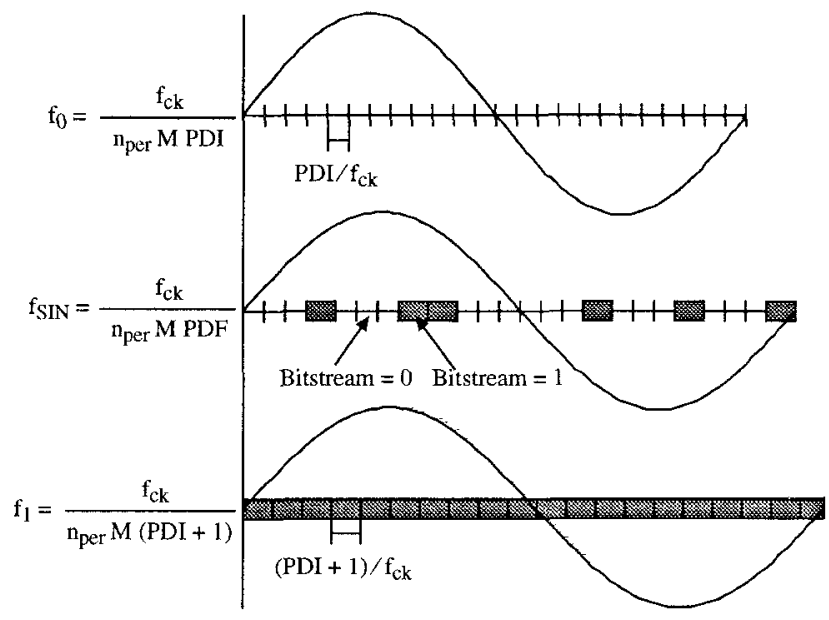

Fig. 4 - Sigma-delta modulation effect

The average frequency of the obtained sinewave, considering a suitable number of master clock periods (at least $2^{13}$ ), is, in fact, very close to the desired value. The simulated frequency error as a function of the desired value, plotted in Fig. 5, demonstrates that the required resolution can be achieved with the proposed approach.

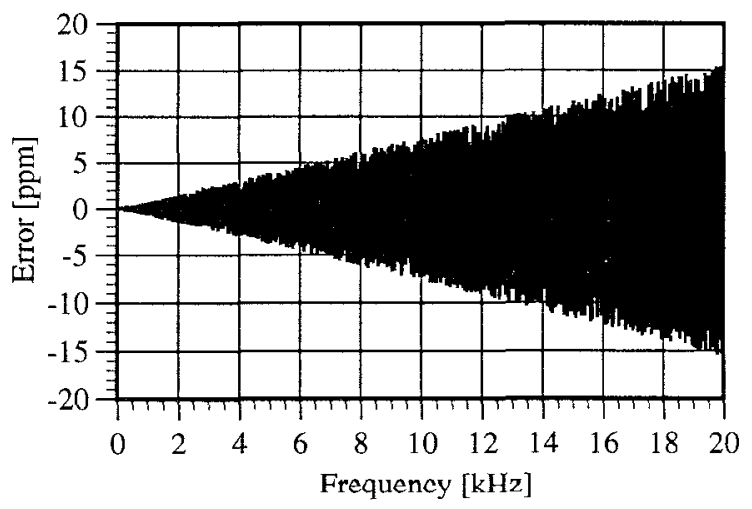

Fig. 5 - Frequency error of the obtained sinewave

Moreover, the resolution obtained at low frequencies is orders of magnitude better than the specifications $\left(\Delta f=15 \mu \mathrm{Hz}\right.$ for $\left.f_{S I N}=100 \mathrm{~Hz}\right)$.

The simulated spectrum of a $2 \mathrm{kHz}$ synthesized sinewave is shown in Fig. 6. It can be observed that the phase noise around the fundamental component due to sigmadelta modulation is well below the required resolution. Moreover, the harmonic distortion introduced by the 10 bit quantization of the digitized sinewave (Sine ROM) is lower than $-70 \mathrm{~dB}$.

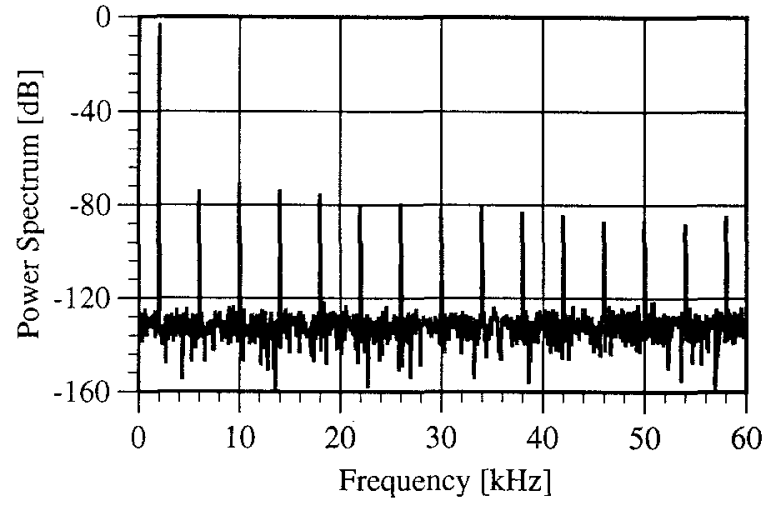

Fig. 6 - Power spectrum of a $2 \mathrm{kHz}$ sinewave

In audiometric applications, when hearing tests are performed in open spaces (without earphones), it is necessary to periodically modulate the frequency of the generated sinewave (from $f_{S I N}$ to $0.9 f_{S I N}$ and back in $200 \mathrm{~ms}$ with 100 steps), in order to avoid stationary waves. This function, called "warble" (Fig. 3), can be easily implemented with the proposed DDS technique. The detailed schematic diagram of the "warble" circuit is shown in Fig. 7. It consists of a shift register, which determines the step amplitude according to

$$
\triangle P D F_{W B}=\frac{f_{c k}}{n_{p e r} M} \frac{10^{-3}}{f_{S I N}\left(1-10^{-3}\right)}
$$

and a digital accumulator, which increments at each time step ( $1 \mathrm{~ms}$ ) the value added to $P D F$ giving rise to the required frequency shift.

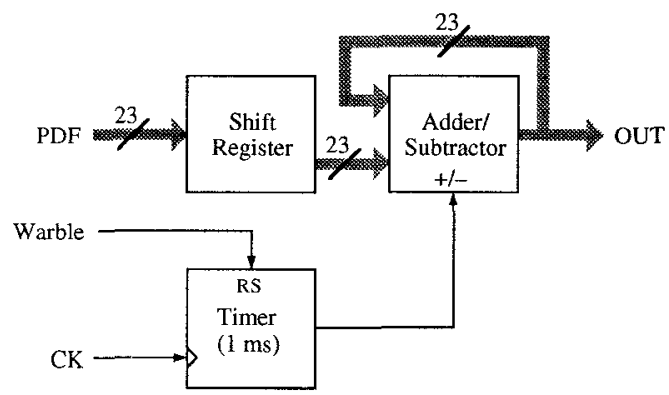

Fig. 7 - Schematic diagram of the "warble" circuit

\section{White NoISE GeNERATOR}

Noise sources are the second family of audio signals required in audiometric tests. Several noise signals, such as white noise, pink noise, narrow-band noise or speech noise, used as masking sounds, can be derived by properly amplifying and filtering a good quality white noise source.

Therefore, one of the most critical issues in the design of noise generators is the choice of the primary white noise source. Analog instruments are typically based on high value resistors operating as white noise sources.

To avoid the drawbacks associated with high-value resistors, we used digital synthesis techniques. This has been accomplished by means of pseudo-random sequence gen- 
erators (PRSG) [5]. For effective audiometric tests a minimum sequence repetition period of $5 \mathrm{~s}$ is required. Therefore, assuming a clock frequency of $32 \mathrm{kHz}$, the random sequence has to be at least $1.6 \times 10^{5}$ samples long, which corresponds to 18 bits. Such a maximal-length sequence can be generated implementing the primitive polynomial

$$
\phi(x)=1 \oplus x^{7} \oplus x^{18} .
$$

The 18 bit output word $(N)$ is then digitally processed in order to reduce the total length to 10 bits, as required by the available DAC. However, a simple truncation of the output word produces a low-pass filtering effect which degrades the quality of the white spectrum. To overcome this problem a compensating high-pass filtering has to be performed. The output word (OUT), therefore, becomes

$$
\text { OUT }=N[9: 0]+N[18: 10]\left(1-z^{-1}\right) .
$$

The Verilog HDL model of the designed PRSG is reported in Fig. 8.

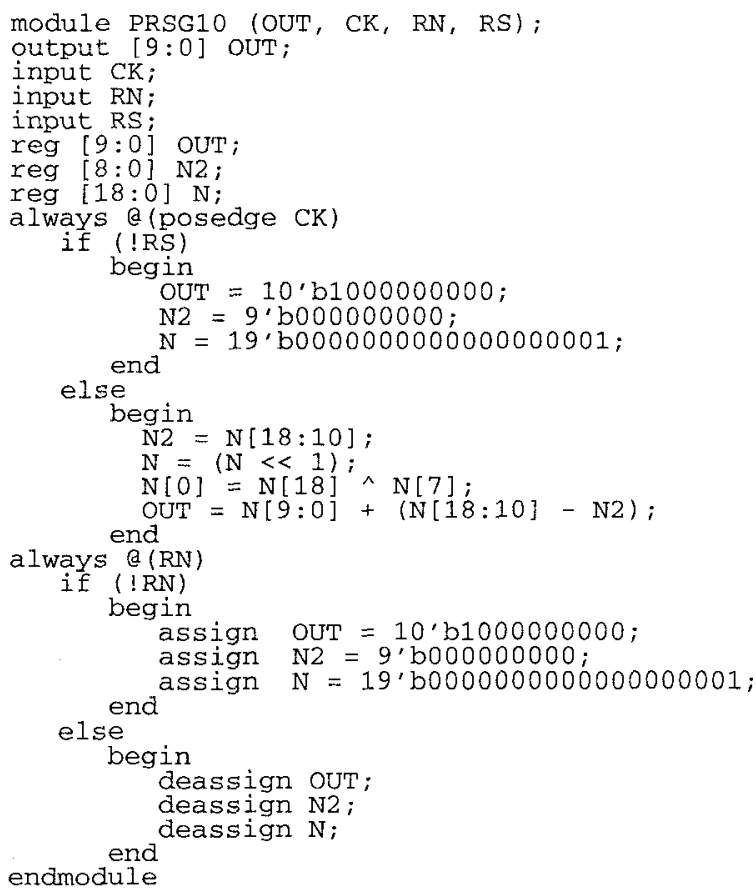

Fig. 8 - Verilog HDL model of the pseudo-random sequence generator

Fig. 9 shows the power spectrum of the white noise signal obtained by simulating the synthesized netlist of the circuit. It can be observed that the flatness of the spectrum is better than $\pm 1 \mathrm{~dB}$.

\section{SYSTEM IMPLEMENTATION}

A prototype implementing the proposed techniques was integrated in a $0.8 \mu \mathrm{m}$ double-poly double-metal CMOS technology. The signal generators (pure tone and white noise) as well as a microprocessor interface were designed using the Verilog HDL description language and synthe-

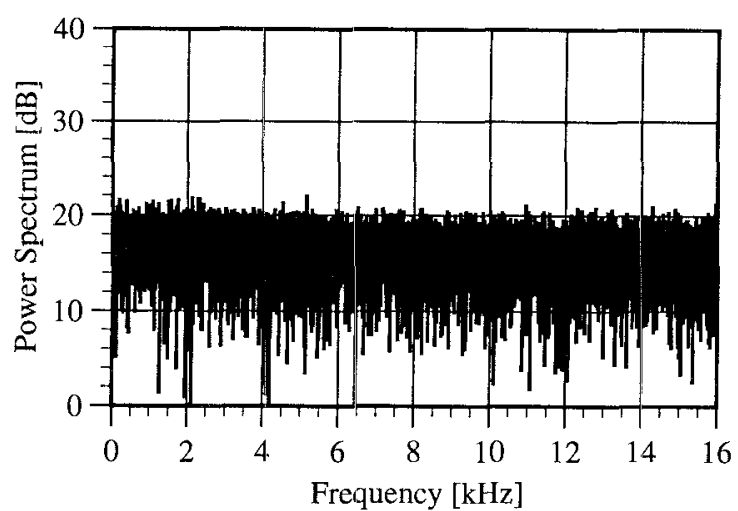

Fig. 9 - Power spectrum of the generated white noise

sized using Synergy. The layout of the chip is shown in Fig. 10. The core area of the complete digital section is $3 \mathrm{~mm}^{2}$.

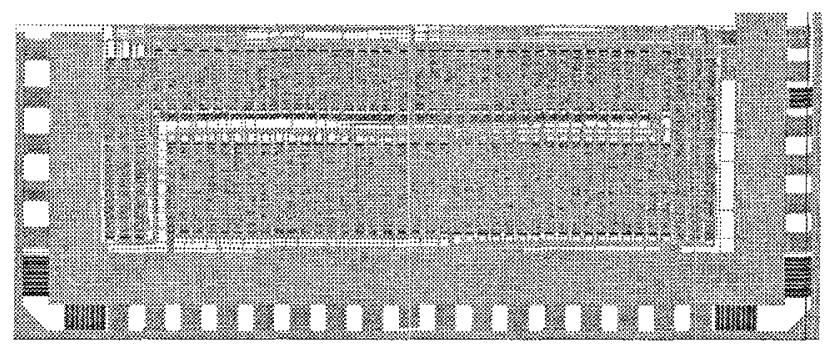

Fig. 10 - Layout of the chip

\section{Conclusions}

In this paper we presented novel digital synthesis techniques for generating variable frequency sinewaves and noise signals for audiometric applications. Simullation results demonstrated that a frequency resolution as low as $15 \mu \mathrm{Hz}$ at $100 \mathrm{~Hz}$ and $1 \mathrm{~Hz}$ at $20 \mathrm{kHz}$ with extremely low phase noise can be achieved. Moreover, a white noise signal with spectral flatness better than $\pm 1 \mathrm{~dB}$ was obtained. A prototype was integrated in a $0.8 \mu \mathrm{m}$ CMOS process.

\section{REFERENCES}

[1] J. C. Candy and A.-N. Huynh, "Double Interpolation for Digital to Analog Conversion", IEEE Trans. on Comm., Vol. 34, No. 1, Jan. 1986, pp. 77-81.

[2] IEC 645-1 and IEC 645-4 Normatives.

[3] H.T.Nicholas III and H. Samueli, "A 150-MHz, Direct Digital Frequency Synthesizer in 1.25- $\mu \mathrm{m}$ CMOS with -90-dBc Spurious Performance", IEEE J. Solid-State Circuits, Vol. SC-26, No. 12, Dec. 1991, pp. 19591969.

[4] P. O'Leary and F. Maloberti, "A Direct-Digital Synthesizer with Improved Spectral Performance", IEEE Trans. on Comm.., Vol. 39, No. 7, July 1991, pp. 1046-1048.

[5] V. N. Yarmolik and I. V. Kachan, Self-Testing.VLSI Design, Elsevier Science Publishers, Amsterdam, The Netherlands, 1993. 